\title{
STAT3 Regulates the Pathogenesis and Bone Mesenchymal Stem Cells Transplantation of Spina Bifida Aperta Through Caspase-8
}

mingyu Jiang ( $\sim 94178268 @ q q . c o m$ )

Harbin Medical University

Ji-cheng Dai

Harbin Medical University

Ming-ying Yin

Harbin Medical University

Ming-yong Ren

Harbin Medical University

$\mathrm{Nan} \mathrm{Wu}$

Harbin Medical University

\section{Ying Gao}

Harbin Medical University

\section{Research}

Keywords: Mesenchymal stem cell, Spina bifida aperta, Signal transducer and activator of transcription 3, Caspase-8, Transplantation

Posted Date: August 4th, 2020

DOl: https://doi.org/10.21203/rs.3.rs-49232/v1

License: (c) (1) This work is licensed under a Creative Commons Attribution 4.0 International License. Read Full License 


\section{Abstract}

Objective: To investigate the influence of signal transducer and activator of transcription-3 (STAT3) on spinal cord tissue grafts of rat fetuses with spina bifida aperta. In particular, we wished to determine if STAT3 would be related to the pathogenesis of spina bifida aperta (SBA) and permit increased survival of spinal cord transplants to improve therapeutic efficiency of cellular transplantation from 20-day pregnant (E20) rats.

Method: Spina bifida aperta were induced with a single intragastric retinoic acid $(140 \mathrm{mg} / \mathrm{kg}$ body weight) administration on E10. STAT3 and caspase-8 expression, caspase-8 positive cells by immunofluorescence on 14, 15, 16 and 17 day in spinal cord of rat fetuses with control group and spina bifida aperta group are analysed. The pregnant rats received fetal surgery and microinjection of Mesenchymal Stem Cells (MSCs) after STAT3 transfection on 16-day pregnant (E16), 17-day pregnant (E17) and 18-day pregnant (E18), P0, P1-6 and to P7-12 of cell passages as well as different injected cell number, then sacrificed on 20-day pregnant (E20) for spine sample collection. The Number of each group was not less than seven. The spinal cord samples were collected directly to detect survival rates of MSCs and caspase-8 expression.

Results: The developmental change in caspase-8 expression of spina bifida aperta was notably increased to the top on E15 compared with no SBA fetuses with Retinoic Acid. STAT3 expression in SBA rat fetuses gradually decreased with embryonic development between E14 and E15, E15 dropped down to bottom. Specifically, the number of caspase-8 positive cells on E15 in spinal cord with SBA rat fetuses was the most; and from E16, the positive cells began to decrease. Compared with STAT3 non-transfection group, MSCs combined with STAT3 transfection on E18, P7-12 and medium injection cell number can statistically improve the success rate of transplantation. In addition, caspase-8 mRNA and protein levels were significantly decreased in STAT3 transfection transplantation contrast for SBA of cellular culture medium and STAT3 non-transfection transplantation.

Conclusions: STAT3 may be associated with the pathogenesis of spina bifida aperta. Furthermore, MSCs transplantation after STAT3 transfection can increase survival rates and reduce apoptosis in the spinal column through caspase-8.

\section{Introduction}

Neural tube defects (NTDs) can lead to serious birth defects in children. At present, it is believed that the main mechanism of NTDs is abnormal closure of neural tube. Among them, congenital spina bifida aperta is the most common [1]. The etiology and pathogenesis of SBA are not clear, and it is generally believed that a variety of abnormal gene regulation and environmental harmful factors take part in the process of embryonic development [2]. Apoptosis is considered to be an important event in the formation, development and maturity of nervous system [3]. Many data show that neural tube malformation in children is related to neuronal apoptosis, but the exact mechanism is still unclear. Developmental biology 
research shows that apoptosis occurs at a specific time and place during the normal development of embryo. If this fine regulated, programmed cell death changes, it may lead to various types of congenital malformations [4].

At present, there is no effective treatment for SBA in children. The severe malformations die in the embryonic period. Even if the children with slight malformation can survive, most of them show neurological dysfunction in different degree, including limb paralysis, defecation dysfunction, paraplegia, etc [5]. It seriously affect the growth and development of children and life quality in the future. However, in recent years, the technology of stem cell transplantation has brought vitality and hope for the treatment of congenital nervous system (NS) malformation. Bone marrow mesenchymal stem cells (BMSCs), as a kind of adult stem cells, have the ability of self-renewal and multi-directional differentiation. Under the action of some physical, chemical factors and cytokines, MSCs can differentiate into neural cells, osteoblasts, chondrocytes, myoblasts, adipocytes and other kinds of cells [6, 7]. It can also differentiate into mesoderm stromal cells, ectoderm or endoderm cells under certain conditions or microenvironment, which plays an important role in the treatment of the nervous system degenerative diseases, stress injury and congenital malformation. This technology is widely used in gene therapy and bioremediation [8, 9]. Because of simple material, easy separation and purification, MSCs still have strong differentiated ability after several passages, and they derive from their own without matching, so immune exclusion is rare. Therefore, MSCs can be used as an important source of seed cells in tissue engineering. MSCs transplantation is expected to be a new method to treat children's SBA.

STAT3 (signal transducer and activator of transcription-3), as an important member of STAT family, was found and purified in 1994 as an acute stage response factor (aprf) [10]. STAT3 can be activated by many cytokines and growth factors, such as IL-6, LIF, CNTF, Onco-statin M and EGF. STAT3 signal

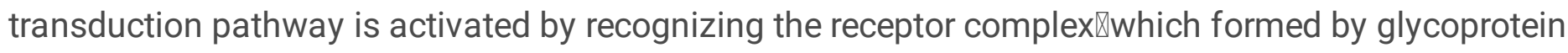
gp130 on cell surface and other molecules such as LIFR and CNTFR. Some scholars believe that the coordination between STAT3 and MAPK signaling pathway plays an active role in maintaining selfrenewal and differentiation of stem cells [11]. STAT and MAPK family are participants of important signal transduction pathways within cells, both of them can transmit stimulation from extracellular to nuclear, and participate in a series of physiological processes such as cell growth, development, differentiation and apoptosis [12]. In addition, STAT3 protein not only participates in many physiological processes in the development of nervous system, but also regulates the survival and repair of nerve cells by neuroregulatory cytokines. Caspase-8 is a major member of caspase family, as a group of aspartic acid specific cysteine protease, which plays a key role in the execution of apoptosis and determines cell morphological and physicochemical change [13]. Meanwhile, it directly involves in early initiation, signal transmission and late effect of apoptosis [14]. Once the expression of caspase-8 is abnormal, it can lead to apoptosis and proliferation disorders, thus inducing occurrence of some nervous system diseases [15].

In this experiment, BMSCs were isolated and purified by using the whole bone marrow adherence method, green fluorescent protein (GFP) was transfected into BMSCs by adenovirus and STAT3 plasmid, and the 
fetal rat model of SBA was established by retinoic acid. The fetal rats were treated with BMSC transplantation by combination of fetal surgery and microsurgery injection. STAT3 and caspase-8 expression in the spinal cord of fetal rats was observed, in order to explore the role of STAT3 in the pathogenesis and transplantation therapy of SBA.

\section{Materials And Methods}

\section{Ethical approval}

All experimental procedures for animal usage were in accordance with the guidelines for care and use of laboratory animals and were approved by the Institutional Animal Care and Use Committee of Harbin Medical University.

\section{Animal models}

Ten- to twelve-week-old adult wistar rats, weighting 250-300g, were selected, which obtained from Animal Research Center of Harbin Medical University (Harbin, China).

Virginal female rats were mated with males of the same strain and the date of vaginal plug detection was designated E0. Spina bifida aperta were induced with a single intragastric retinoic acid $(140 \mathrm{mg} / \mathrm{kg}$ body weight; Sigma) administration on E10 as previously described (Cai, W.; Zhao, H.; Guo, J.; Li, Y.; Yuan, Z.; Wang, W. Retinoic acid-induced lumbosacral neural tube defects: myeloschisis and hamartoma. Childs Nerv. Syst. 23(5):549-554; 2007). Spinal cord specimens of fetal rats were obtained from E14, E15, E16 and E17.

\section{Isolation and transfection of bone marrow mesenchymal stem cells}

The cells were obtained from the femur of 4-weeks old wistar rats. Rat bone marrow was flushed with $20 \mathrm{ml} \mathrm{DMEM} / \mathrm{F} 12$ medium and 10\% fetal bovine serum (FBS), and cell suspension was isolated as MSCs through the method of bone marrow adherence for the primary generation (P0), then cells were seeded in a tissue culture incubator at $37^{\circ} \mathrm{C}$ under $5 \% \mathrm{CO}$. Cells were passaged 5-7 days to achieve the desired dose until passage 12 (P12). The MSCs of P12 were plated in a 6-well plate to grow to $90 \%$ confluent before transfection (about $1 \times 10^{5}$ cells/well). Twenty four hours before transplantation, MSCs were transfected with eGFP expression adeno-5 vector (SinoGenoMax.Co., Ltd, Beijing, China)(100pfu/1cell). Productive infection were checked by observing Green fluorescent protein (GFP) expression under the quantitative real-time PCR assay. Adenovirus carrying green fluorescence protein (Ad-EGFP) and STAT3 plasmid (Invitrogen Co, San Diego, USA) was constructed according to the above method. The concentration of EGFP-MSCs suspension for transplantation is about 20000 cells $/ \mu$ l.

\section{Transplantation procedure and animal group}

A total of seventy-two female wistar rats were subjects of this study. Animals had free access to food and water throughout the study. On embryonic day (E16, E17 and E18), all the surgeries were done under 
anesthesia with a combination of Chloral Hydrate $(10 \%, 300 \mathrm{mg} / \mathrm{kg})$ and atropine $(0.25 \mathrm{mg} / \mathrm{ml})$ intraperitoneally. Then the pregnant rats underwent midline laparotomy to expose the uterine horns. To relieve uterine spasm, the uterus was covered with wet gauze immersed with warm physiologic saline and atropine $(0.1 \mathrm{mg} / \mathrm{kg})$ was given intraperitoneally. Under the microscope the position of lumbosacral spine of fetus was identified through the wall of uterus. Then 7-0-nylon purse-string suture and a small incision were made on the wall of uterus. The amniotic sac was opened and the defective region of spinal cord was exposed. eGFP transfected MSCs suspension $(0.2 \mu \mathrm{l} /$ injection site) was injected into the defective region of spinal cord with a micropipette (internal tip diameter $100 \mu \mathrm{m}$ ) connected to a Hamilton syringe. Micropipettes for injection were made from borosilicate glass capillaries (model GD-1; Narishige Scientific Instruments, Tokyo, Japan) by using a micropipette puller (model PB-7; Narishige Scientific Instruments, Tokyo, Japan). After MSCs injection, the fetuses were returned to the uterus, and the wound of the uterus was closed. The same volume of MSCs free culture medium and STAT3 non-transfection was injected as control group (blank group). In average, 2 to 3 fetuses could be injected in one dam without compromising the survival of the fetuses. The pregnant rats recovered from the anesthesia within 1 hour and were returned to their home cage. The pregnant rats on E20 were reanesthetized with an overdose of pentobarbitone sodium, and the fetuses that had been transplanted were harvested by cesarean section. Each fetus was inspected for the presence of external malformations.

\section{Real-time polymerase chain reaction analysis}

Total RNA was isolated using TRIzol reagent (Invitrogen Co, USA) according to the manufacturer's instructions, and cDNA was generated through reverse transcription using the PrimeScript RT Master Mix kit (TaKaRa Bio Inc, Japan). Equal amounts of cDNA were diluted and amplified through real-time polymerase chain reaction (PCR) using All-in-one qPCR Mix (TaKaRa Bio Inc) in a $20 \mu$ reaction volume containing $10 \mu \mathrm{l}$ of $2 \times$ All-in-one qPCR Mix (TaKaRa Bio Inc), $1 \mu \mathrm{l}$ of $2 \mathrm{mmol} / \mathrm{L}$ forward primer, $1 \mu \mathrm{l}$ of 2 $\mathrm{mmol} / \mathrm{L}$ reverse primer, $1 \mu \mathrm{l}$ of $\mathrm{CDNA}$, and $6 \mu \mathrm{l}$ of nuclease-free water. After an initial denaturation step for $10 \mathrm{~min}$ at $95^{\circ} \mathrm{C}$, the conditions for cycling were 40 cycles of $10 \mathrm{~s}$ at $95^{\circ} \mathrm{C}, 20 \mathrm{~s}$ at $60^{\circ} \mathrm{C}$, and $15 \mathrm{~s}$ at $72^{\circ} \mathrm{C}$. For the normalization of each sample, glyceraldehyde 3-phosphate dehydrogenase (GAPDH, TaKaRa Bio Inc) primers were used to measure the amount of GAPDH cDNA. The primers used are listed below: GAPDH: sense: 5'-TCGCCAGCCGAGCCACAT-3', antisense: 5'-GGAACATGTAAACCATGTAGTTG-3'; STAT3: sense: $5^{\prime}-$ GCCAG

AGAGCCAGGAGCA-3', antisense: 5'-ACACAGATAAACTTGGTCTTCAGGTATG-3'; caspase-8: sense: 5'TGCAAATGAAATCCACGAGA-3', antisense: 5'- CCAGGTTCCATTCACA

GGAT-3'. The relative fold-change in the target gene cDNA was determined using the $2-\Delta \Delta \mathrm{Ct}$ method.

\section{Western blotting analysis}

The protein levels in spinal cord tissues were detected by Western blotting analysis. Briefly, the tissues from different groups were lysed by Radio-Immunoprecipitation Assay lysis buffer (Beyotime Bio Inc, Shanghai, China). The protein concentrations were determined by BCA protein estimation kit (Beyotime 
Bio Inc). Then, equal amounts of protein samples were separated by sodium dodecyl sulphatepolyacrylamide gel electrophoresis (Beyotime Bio Inc), and transferred to polyvinylidene difluoride membranes (Beyotime Bio Inc). After blocking with 5\% non-fat dry milk, the membranes were incubated with primary antibodies at $4^{\circ} \mathrm{C}$ overnight. The primary antibodies used in this study were as follows: STAT3 (Santa Cruz Bio Inc, USA), caspase-8 (Santa Cruz Bio Inc, USA), and GAPDH (Santa Cruz Bio Inc, USA). Then the corresponding secondary antibodies conjugated with horseradish peroxidase were added. ECL detection reagent (7 Sea Biotech, Shanghai, China) was used to visualize the blots. The results were quantified with Gel-Pro-Analyzer (Media Cybernetics, USA).

\section{Immunofluorescence}

To determine the percentage of MSC differentiation and survival, all GFP immuno-positive cells were immuno-positive for the neuron/glia marker on each section were counted. For cell staining, microscopic slide with MSCs seeded $24 \mathrm{hrs}$ before were fixed with $4 \%$ paraformaldehyde for $30 \mathrm{~min}$., and were analysed by immunofluorescence. Ten (40xmagnification) non-contiguous and non-overlapping random chosen microscopic fields distributed evenly across the slide were examined, the number of MSCs expressing the specific neuron/glia marker was counted by observers who were not informed of the identities of the experiments. The percentages of MSCs expressing specific marker were determined and reported as the number of positive cells/total cells.

\section{Statistical analysis}

All analyses were performed in a double-blind manner. All values are expressed as mean \pm SEM. Student's t-test and Bonferroni's test wereused for single and multiple comparisons, respectively. Chi-square test was used for comparisons of foetuses survival rates. $P$ values less than 0.05 were considered to be statistically significant.

\section{Results}

\section{Survival of rat fetuses in each group before and after MSCs transplantation}

267 spinal cord specimens of fetal rats were obtained, including 47 cases of no SBA formation with Retinoic Acid (E14, 11 cases; E15, E16 and E17, 12 cases per group), 48 cases of SBA formation with RA (E14, E15, E16 and E17, 12 cases per group), The groups above aren't received fetal surgery; Seventy-two pregnant rats were MSCs transplanted, eight of them died after transplantation on the 18th day, the survival rate of transplanted pregnant rats was $89 \%$ (64/72). 24 cases belonged to transplantation of cellular culture medium into SBA, 76 cases from MSCs transplantation into SBA with STAT3 plasmid transfection and 72 cases with non-STAT3 transfection.

\section{Analysis of STAT3 and caspase-8 expression on 14, 15, 16 and 17 day in spinal cord of rat fetuses with control group and spina bifida aperta group}


The developmental change in caspase-8 expression of spina bifida aperta was notably different from the change that occurred in no SBA fetuses with Retinoic Acid, especially at embryonic day15 (E15).

Compared with controls, both caspase- 8 mRNA and protein levels were significantly increased in spina bifida rat fetuses between E14 and E15, and E15 reached the peak. However, with no significant difference at E16 or E17. Overall, the expression of STAT3 mRNA and protein levels in SBA rat fetuses gradually decreased with embryonic development between E14 and E15, E15 dropped down to bottom. Then From E16 to E17, STAT3 expression increased more steadily in SBA rat fetuses than that in no SBA fetuses with Retinoic Acid (Fig.1 A-E).

\section{Analysis of caspase-8 positive cells on 14, 15, 16 and 17 day in spinal cord of rat fetuses with control group and spina bifida aperta group by immunofluorescence}

From E14 to E17, the average number of caspase-8 positive cells in spinal cord with SBA rat fetuses was more than that in control group at the same time, and the fluorescence intensity was higher than that in control group, which was statistically significant $(P<0.05)$. Specifically, the number of caspase-8 positive cells on E15 in spinal cord with SBA rat fetuses was the most; and from E16, the positive cells began to decrease (Fig.2). In addition, the number of caspase-8 positive cells in spinal cord with SBA rat fetuses on E15 and E16 was significantly higher than that on E14 or E17, which was also statistically significant $(P<$ $0.05)$.

\section{MSCs combined with STAT3 transfection can improve the success rate of transplantation}

Before transplantation, we found that MSCs displayed fibroblast-like, spindle-shaped morphology from control group in culture. MSCs in culture was examined by CD44 expression. The purity of MSCs was enriched from $45 \pm 1.02 \%$ at $P 0$ to $55 \pm 1.22 \%$ at $P 1-6$, and $60 \pm 1.16 \%$ from P7 to P12. No obvious morphological changes were observed within the culture period. However, MSCs combined with STAT3 transfection also processed as above. We observed that the cells were long fusiform, round, elliptical, flat, irregular shapes and so on (Fig.3). The purity of MSCs was enriched from $75 \pm 1.13 \%$ at P0 to $85 \pm 1.07 \%$ at P1-6, and $91 \pm 0.29 \%$ from P7 to P12 when CD44 expression was also tested, which was statistically significant ( $(P=0.03,0.023$ and 0.017 compared with non-transfection group, respectively). Then we transplanted MSCs into each group of rat fetuses on E16, E17 and E18 to examine whether STAT3 transfection may affect cell survival ratio. The pregnant, transplanted rats were killed on E20, then rat fetuses were harvested for lumbosacral spinal sample collection. Firstly, The overall survival rates of MSCs after STAT3 transfection was found to be $36 \pm 1.08 \%$ on E16, $42 \pm 0.93 \%$ on E17 and $46 \pm 0.72 \% \mathrm{E} 18$, which was a little higher than that of non-transfection group (19 $\pm 0.83 \%$ on $\mathrm{E} 16,21 \pm 0.92 \%$ on $\mathrm{E} 17$ and $24 \pm 1.07 \%$ E18) and statistically significant ( $(P=0.041,0.033$ and 0.031 compared with non-transfection group, respectively). Better survival after STAT3 transfection was achieved in the transplanted groups performed on later development stages (E18) as compared with E16 and E17 group, but it did not reach a statistical significance ( $P=0.65 E 16$ versus $E 18 ; P=0.32 E 17$ versus $E 18)$. Secondly, we wanted to detect whether different MSC passages transplanted after STAT3 transfection could interpose survival ratio and three groups were divided on E20 from rat fetuses: P0, P1-6 and to P7-12. MSCs survival was found to be 
P0 (41.09 $\pm 1.2 \%), \mathrm{P} 1-6(43.36 \pm 0.8 \%)$ and P7-12 (44.06 $\pm 0.8 \%)$, which showed a notable increase compared with P0 (20.21 $\pm 0.9 \%), \mathrm{P} 1-6(21.36 \pm 1.1 \%)$ and P7-12 (22.01 $\pm 0.7 \%)$ of non-transfection group, again the differences reached a statistical significance $(P=0.026,0.021$ and 0.023 , respectively). Thirdly, we evaluated the effects of injected cell number on survival ratio after STAT3 transfection on E20 from rat fetuses. Medium injection group (2000-4000 cells/injection/spinal cord, $45.92 \pm 1.3 \%$ ) was remarkably different from the change that occurred in low injection group (1000-2000 cells/injection/spinal cord, $42.85 \pm 0.9 \%)$ and high injection group (4000-6000 cells/injection /spinal cord, $42.32 \pm 0.6 \%$ ), which reached a statistical significance $(P=0.029,0.026$ and 0.032 , respectively) compared with low injection group $(21.12 \pm 0.7 \%)$, medium injection group $(22.87 \pm 0.9 \%)$ and high injection group $(20.98 \pm 0.8 \%)$ of nontransfection group.

\section{Analysis of caspase-8 expression in spinal cord of each group after MSCs transplantation}

Caspase-8 as apoptosis gene plays an important role in the development of spina bifida aperta as before. Next we studied the apoptosis of whole spinal column after STAT3 transfection transplantation by caspase-8 expression. Compared with SBA of cellular culture medium and STAT3 non-transfection transplantation, both caspase-8 mRNA and protein levels were significantly decreased in STAT3 transfection transplantation on E16 $(P=0.035,0.029)$, similar to $\mathrm{E} 17(\mathrm{P}=0.031,0.028)$ and $\mathrm{E} 18(\mathrm{P}=0.029$, 0.026) with a statistical significance (Fig.4 A-C). However, the comparison of caspase-8 expression from E16 to E18 after STAT3 transfection did not reach a statistical significance ( $P=0.67$ E16 versus E17; $P=$ $0.35 \mathrm{E} 16$ versus $\mathrm{E} 18)$.

\section{Discussion}

Neural tube defects (NTDs) are a class of congenital malformations with serious consequences. The worldwide incidence of NTDs ranges from 1.0 to 10.0 per 1000 births [16]. Almost equal frequencies between two major categories: anencephaly and spina bifida aperta [17]. The exact mechanism of NTDs is unknown up to now, a variety of genetic and environmental factors are implicated [18]. Besides, The development in nervous system is a complex process that involves the proliferation, differentiation or apoptosis of neurons, glial cells synapse, neural networks and nerve nuclei in three-dimensional building. Apoptosis is also called programmed cell death, which is a special form of cell death distinguished from necrosis. It is subject to its internal organization of certain cells in the gene-regulation and active biological phenomenon suicide, which is widespread in embryonic development, organ or tissue morphogenesis [19]. The regulation from the nervous system, neurotrophic factors secreted by cells and other mechanisms restrains excessive cell apoptosis. If any element of these mechanisms is damaged, it will result in excessive cells apoptosis to the occurrence of NTDs [20]. Genes contributing to NTDs in animal models provide important candidate information for risk association study in human malformations. SNPs in genetic loci including SHH, BMP4, NOG, CCL2, PRKACA, PRKACB, NAP1L2 and TERC, have been confirmed for risk association with humans NTDs [21]. The defects occur in the transformation from neural groove closure to neural tube. Anencephalus takes place in the formation of neural groove non-closure, and the closed defect in the middle or end of neural groove lead to the spina 
bifida aperta or other malformations [22]. The reasons for neural tube defects are in different opinions, some think that the closure of neural tube stops in embryonic period, others claim that the excessive cerebrospinal fluid makes ventricular pressure increase remarkably, leading to re-open of the closed neural tube [23]. However, the exact mechanism of neural tube defects is not clear, generally considered to the common results of genetic and environmental factors.

Mammalian embryonic development is a highly dynamic process involving a high degree of embryonic cellular division, growth, and differentiation which can be

influenced by disrupted signaling pathways (Wells, Lee, McCallum, Perstin, \& Harper, 2010). STAT3 regulates multiple embryonic development pathways that play an important role in the abnormal embryonic development which may be altered by VPA [24]. In addition, STAT3 modulates gene transcription in response to intermediate factors, such as EGF, IL- 6 and caspase-8, whereas these factors regulate cell proliferation, survival, and apoptosis during embryogenesis (Hirano, Ishihara, \& Hibi, 2000; Liu et al., 2010; Zhong et al., 1994). Caspase-8 is a key promoter in the apoptosis pathway. It occupies a central position in the process of apoptosis and directly participates in early initiation, signal transmission and late-phase reaction [25]. The abnormal expression of caspase-8 can lead to apoptosis and proliferation disorder, thus inducing occurrence of some diseases, such as spina bifida aperta [26]. In this study we report that both caspase-8 mRNA and protein levels increased the peak on E15 in spina bifida rat fetuses, and STAT3 expressions show opposite change at the same time. Meanwhile, the number of caspase-8 positive cells on E15 in spinal cord with SBA rat fetuses was the most by immunofluorescence. From these results we can draw the conclusion that STAT3 plays an important role in the occurrence of spina bifida aperta on E15, and then regulates the process of cell apoptosis through caspase-8 during embryogenesis.

RA in vivo comes from vitamin A in food, which is metabolized to all-trans- RA after absorption by the body. RA receptor (RAR) and retinol receptor (RXR) combined with all-trans- RA can bind to the specific DNA sequence of the target gene and start the transcription. RA is very critical for the development of CNS, Wilson L et al [27] found that lack of RA does not cause spina bifida or encephalopathy. However, Excessive RA can lead to NTD, which is due to up-regulation of receptor, resulting in a large areas of cell apoptosis, and NTD malformation can be observed by morphology. Except for NTD, RA can also cause developmental abnormalities such as sacrococystal insufficiency, craniofacial deformities and limb defects [28]. Down-regulation expression of STAT3 in NTD may be due to induce by RA through its receptor, which affects gene transcription related to cell survival. Once cell survival is inhibited, caspase-8 expression will be up-regulated, and cell apoptosis appearance leads to the occurrence of NTD. Neurogenesis is a highly complex embryological event involving many factors. At the gene level, these processes are the results from a series of genes, expressing and interacting in a highly specific spatiotemporal pattern [29]. STAT3, as a member of signal transducer and transcription activator family, plays an important role. E14 is the time when the sheath cells begin to migrate to the limbic layer. At this time, STAT3 are up-expressed in the spinal cord tissue, suggesting that STAT3 may participate in the differentiation and migration of neural precursor cells. On E15, apoptotic cells with the most positive 
fluorescence were observed in the spinal cord, which indicated that the expression of STAT3 is downregulation after the migration and differentiation of cells. STAT3 has different expression in E14, E15, E16 and E17, which may demonstrate that STAT3 is activated by different growth factors or cytokines in different stages of neurodevelopment and has different biological functions in many aspects.

Mesenchymal stem cells (MSCs) has a strong self-renewal capacity and pluripotency, which could be induced to differentiate into bone cells, cartilage cells, fat cells, endothelial cells, myocytes, nerve cells and other cell types in particular environment [30]. The MSCs transplantation of spina bifida aperta can repair a variety of neuronal and bone abnormalities. In addition, MSCs are easily obtained, operated, cultured in vitro and purified, which can be passaged for many times to maintain differentiation capacity, but no matching and immune rejection. It has recent studies showed that MSCs can survive in the spinal cord injury and differentiate into neurons and astrocytes to reduce the damage caused by the loss of nerve function [31]. Therefore, MSCs can be used as tissue-engineering cells, and MSCs transplantation for the treatment of spina bifida aperta is expected to a most promising method of nervous system malformations. The purposes of surgical repair on spinal cord for treatment early in utero of rat fetuses with spina bifida aperta are to reduce further aggravation of malformations. Our study showed that MSCs combined with STAT3 transfection can remarkably improve cell differentiation morphology and purity. Cell transplantation from P7 to P12 on E18 with medium injection (2000-4000 cells/injection/spinal cord) after STAT3 transfection could obtain better therapeutic effect than SBA with cellular culture medium and non-STAT3 transfection transplantation. both caspase-8 mRNA and protein levels after STAT3 transfection were significantly decreased compared with two control groups. Studies have shown that STAT3 pathway may play an important role in the migration and differentiation of MSCs [32]. STAT3 interacts with other signaling pathways, such as MAPK and PI32K, which is expressed and activated during the development of nervous system, and participates in the differentiation of stem cells in neural tube epithelium [33]. Peripherin, as a neuron specific intermediate filament protein, which has a STAT3 binding site in the promoter and is expressed in all peripheral neurons, sensory and motor neurons [34]. STAT3 plays a transcriptional activity under the action of LIF and IL-6, suggesting that it also has regulatory function on peripheral neuron [35]. Aberg $M$ et al [36] observed that STAT3 activation can specifically mediate the directional differentiation of type II astrocytes. All these results suggest that JAK/STAT signaling pathway activated by cytokine is an important mechanism in determining the direction of cell differentiation during mammalian development. Although STAT3 is one of the most important regulatory genes in the development of nervous system, the survival rate of embryos after gene knockout is very low in vivo, which limits STAT3 research on the regulation process and molecular mechanism [37]. In recent years, the culture success of MSCs in vitro has provided a good experimental model for further study of the important biological characteristics, such as proliferation, differentiation, migration and so on [38]. Therefore, the effect of STAT3 partial activation on the proliferation and differentiation of MSCs in vitro has not been reported. JAK/STAT3 signaling pathway is closely related to maintain pluripotency and undifferentiated state of embryonic stem cells [39]. Under LIF treatment, stem cells overexpressing dominant negative STAT3 will lose the ability of self-renewal and differentiate, revealing that STAT3 is closely related to the proliferation of neural stem cells [40]. Our results showed 
that the proliferation of MSCs transfected with STAT3 plasmid was vigorous, while the cells without STAT3 plasmid transfected grew slowly and the number of cells decreased. It is suggested that the activation of STAT3 does affect the proliferation of MSCs. On the one hand, it can accelerate the growth of MSCs; on the other hand, it may increase survival and proliferation of cells. In addition, some studies show that STAT3 plays an important role in the differentiation of neural precursor cells in vivo and interacts with other signaling pathways, such as MAPK and caspase-8, and then participates in neuromodulatory cytokine (NF), leukemia inhibitory factor (LIF), ciliary neurotrophic factor (CNTF), CT1 (cardiotrophin), Oncostatin $M$ to regulate the differentiation and fate of neural precursor cells [41]. In this process, SOCS family, as STAT3 inhibitors, acts as a negative feedback protein to inhibit JAK activity. Moreover, STAT3 has a binding site in the promoter of glial fibrillary acidic protein (GFAP), and activates the transcription of GFAP during the differentiation of cortical precursor cells into glial cells induced by CNTF. Therefore, it is believed that STAT3 is closely related to the differentiation of astrocytes [42]. Further studies showed that STAT3 combined with Smad1 and p300 formed a complex named as STAT3/Smad1/p300 through synergistic treatment of LIF and bone morphogenetic protein 2 (BMP-2), which mediated the activation of GFAP promoter and induced primary embryonic neural precursor cells to differentiate into astrocytes. The above-mentioned evidence proved that STAT3 mainly induces the differentiation of neural stem cells into astrocytes [43]. Our results also show that STAT3 is involved in the differentiation of MSCs into osteocytes, muscular and neuroglia cells. STAT3 signaling pathway and MAP kinase mediate neurite growth induced by LIF in PC12 cells. The activation of STAT3 can negatively regulate the neurite elongation of PC12 cells dependent on Ras-MAPK pathway, and promote the contraction of sympathetic neuron dendrites in vitro [44]. It is demonstrated that STAT3 can regulate neurite growth, which is consistent with the results of our studies.

Meanwhile, caspase- 8 plays the role of initiator in the caspase cascade and is a key molecule in death receptor-induced apoptotic pathways. It has been shown that caspase-8 is essential for Fas-mediated apoptotic signaling [45]. Activated caspase-8 cleaves and activates downstream caspases, thereby committing the cell to apoptosis. Caspase-8 also activates another apoptotic pathway, which, through processing

of Bid, mediates release of cytochrome c from mitochondria and activation of caspase-9. And, in addition to Fas, caspase-8 is involved in apoptotic signaling via other death receptors, such as tumor necrosis factor receptor type 1 (TNFR1), death receptor 3 (DR3) and TNF-related apoptosis-inducing ligand (TRAIL) receptors. Clearly, caspase-8 is an important mediator for a wide range of apoptotic pathways [46]. In our study, compared with SBA of cellular culture medium and STAT3 non-transfection transplantation, both caspase-8 mRNA and protein levels were significantly decreased in STAT3 transfection transplantation. The results mean that MSCs transplantation after STAT3 transfection can reduce apoptosis in the spinal column. The caspase-8-mediated signaling pathway, as apoptosis functions during MSCs transplantation after STAT3 transfection, requires further elucidation.

\section{Conclusion}


STAT3 plays an important role in the regulation of cell apoptosis and signal transduction with SBA, as well as the differentiation and transformation of stem cells. Our study also explored the role of STAT3 in MSCs transplantation. In order to better serve the clinical prenatal diagnosis, early treatment of congenital malformation, and improve the quality of life of children with spina bifida aperta, lay a solid foundation, which is an urgent problem to be solved.

\section{Abbreviations}

STAT3: signal transducer and activator of transcription-3; MSCs: mesenchymal Stem Cells; SBA: spina bifida aperta; E20: 20-day pregnant; NTDs: neural tube defects; GFP: green fluorescent protein; RA: retinoic acid; NF: neuromodulatory cytokine; LIF: leukemia inhibitory factor; CNTF: ciliary neurotrophic factor; GFAP: glial fibrillary acidic protein; TNFR1: tumor necrosis factor receptor type 1; TRAIL: TNF-related apoptosis-inducing ligand; MAPK:mitogen-activated protein kinase.

\section{Declarations}

\section{Funding}

This study was supported by Heilongjiang Provincial Universities, projects of basic scientific research business funds, from Education Department of Heilongjiang Province in 2019.

\section{Availability of data and materials}

The datasets used and/or analyzed during the current study are available from the corresponding author on reasonable request.

\section{Author contribution}

Jiang Ming-yu performed all experimental work and data analysis and drafted the manuscript. Wu Nan significantly contributed to all the figure designs used throughout the manuscript. Yin Ming-ying, Ren Ming-yong, and Gao Ying contributed significantly to experimental design and critically reviewed the manuscript. Dai Ji-cheng conceived the concept of work, contributed to experimental design, and reviewed the manuscript.

\section{Ethics approval and consent to participate}

The study is approved by the ethical committee of Harbin medical University of technology committee (approval number HMU-2007).

\section{Consent for publication}

Not applicable

Conflicts of interest 
No competing interests, financial or otherwise, declared by the authors.

\section{References}

[1] Jeelani Y, Mosich GM, McComb JG. Closed neural tube defects in children with caudal regression. Childs Nerv Syst. 2013;29(9):1451-7.

[2] Aoulad Fares D, Schalekamp-Timmermans S, Nawrot TS, Steegers-Theunissen RPM. Preconception telomere length as a novel maternal biomarker to assess the risk of spina bifida in the offspring. Birth Defects Res. 2020,15;112(9):645-651.

[3] Wang S, Ji D, Yang Q, Li M, Ma Z, Zhang S, et al. NEFLb impairs early nervous system development via regulation of neuron apoptosis in zebrafish. J Cell Physiol. 2019;234(7):11208-11218.

[4] Geden MJ, Romero SE, Deshmukh M. Apoptosis versus axon pruning: Molecular intersection of two distinct pathways for axon degeneration.Neurosci Res. 2019;139:3-8.

[5] Abdallah MA, Ahmed KM, Recio-Restrepo MV, Khalid M, Yeddi A, Abu-Heija A, et al. Pneumococcal Meningitis Complicated by Spinal Cord Dysfunction and Acute Polyradiculopathy. Ochsner J. 2020;20(2):219-221.

[6] Kitada M, Dezawa M. Induction system of neural and muscle lineage cells from bone marrow stromal cells; a new strategy for tissue reconstruction in degenerative diseases. Histol Histopathol. 2009;24(5):631-42.

[7] Muench MO, Barcena A. Stem cell transplantation in the fetus. Cancer Control.

2004; 11: 105-18.

[8] Golchin A, Farahany TZ, Khojasteh A, Soleimanifar F, Ardeshirylajimi A. The Clinical Trials of Mesenchymal Stem Cell Therapy in Skin Diseases: An Update and Concise Review. Curr Stem Cell Res Ther. 2019;14(1):22-33.

[9] Myneni VD, McClain-Caldwell I, Martin D, Vitale-Cross L, Marko K, Firriolo JM, et al. Mesenchymal stromal cells from infants with simple polydactyly modulate immune responses more efficiently than adult mesenchymal stromal cells. Cytotherapy. 2019;21(2):148-161.

[10] Hsia HC, Hutti JE, Baldwin AS. Cytosolic DNA Promotes Signal Transducer and Activator of Transcription 3 (STAT3) Phosphorylation by TANK-binding Kinase 1 (TBK1) to Restrain STAT3 Activity. J Biol Chem. 2017;292(13):5405-5417.

[11] Al-Massri KF, Ahmed LA, El-Abhar HS. Mesenchymal stem cells therapy enhances the efficacy of pregabalin and prevents its motor impairment in paclitaxel-induced neuropathy in rats: Role of Notch1 receptor and JAK/STAT signaling pathway. Behav Brain Res. 2019;360:303-311. 
[12] Mossahebi-Mohammadi M, Quan M, Zhang JS, Li X. FGF Signaling Pathway: A Key Regulator of Stem Cell Pluripotency. Front Cell Dev Biol. 2020,18;8:79.

[13] Fritsch M, Günther SD, Schwarzer R, Albert MC, Schorn F, Werthenbach JP, et al. Caspase-8 is the molecular switch for apoptosis, necroptosis and pyroptosis. Nature. 2019;575(7784):683-687.

[14] Schwarzer R, Laurien L, Pasparakis M. New insights into the regulation of apoptosis, necroptosis, and pyroptosis by receptor interacting protein kinase 1 and caspase-8. Curr Opin Cell Biol. 2020;63:186-193.

[15] Yin F, Zhou H, Fang Y, Li C, He Y, Yu L, et al. Astragaloside IV alleviates ischemia reperfusion-induced apoptosis by inhibiting the activation of key factors in death receptor pathway and mitochondrial pathway. J Ethnopharmacol. 2020,10;248:112319.

[16] Auffret M, Cottin J, Vial T, Cucherat M. Clomiphene citrate and neural tube defects: a meta-analysis of controlled observational studies. BJOG. 2019;126(9):1127-1133.

[17] Zou J, Wang F, Yang X, Wang H, Niswander L, Zhang T, et al. Association between rare variants in specific functional pathways and human neural tube defects multiple subphenotypes. Neural Dev. 2020,10;15(1):8.

[18] Zohn IE. Mouse Models of Neural Tube Defects. Adv Exp Med Biol. 2020;1236:39-64.

[19] Zhang L, Dong Y, Wang W, Zhao T, Huang T, Khan A, et al. Ethionine Suppresses Mitochondria Autophagy and Induces Apoptosis via Activation of Reactive Oxygen Species in Neural Tube Defects. Front Neurol. 2020,7;11:242.

[20] Wei X, Cao S, Ma W, Zhang C, Gu H, Liu D, et al. Intra-Amniotic Delivery of CRMP4 siRNA Improves Mesenchymal Stem Cell Therapy in a Rat Spina Bifida Model. Mol Ther Nucleic Acids. 2020,5;20:502-517.

[21] Wu J, Bao Y, Lu X, Wu L, Zhang T, Guo J, et al. Polymorphisms in MTHFD1 Gene and Susceptibility to Neural Tube Defects: A Case-Control Study in a Chinese Han Population with Relatively Low Folate Levels. Med Sci Monit. 2015,4;21:2630-7.

[22] Li X, Yuan Z, Wei X, Li H, Zhao G, Miao J, et al. Application potential of bone marrow mesenchymal stem cell (BMSCs) based tissue-engineering for spinal cord defect repair in rat fetuses with spina bifida aperta. J Mater Sci Mater Med. 2016;27(4):77.

[23] Xue J, Gu H, Liu D, Ma W, Wei X, Zhao L, et al. Mitochondrial dysfunction is implicated in retinoic acidinduced spina bifida aperta in rat fetuses. Int J Dev Neurosci. 2018;68:39-44.

[24] Li H, Zhang Z, Gao C, Wu S, Duan Q, Wu H, et al. Combination chemotherapy of valproic acid (VPA) and gemcitabine regulates STAT3/Bmi1 pathway to differentially potentiate the motility of pancreatic cancer cells. Cell Biosci. 2019,18;9:50. 
[25] Tummers B, Green DR. Caspase-8: regulating life and death. Immunol Rev. 2017;277(1):76-89.

[26] Wu Y, Wang F, Reece EA, Yang P. Curcumin ameliorates high glucose-induced neural tube defects by suppressing cellular stress and apoptosis. Am J Obstet Gynecol. 2015;212(6):802.e1-8.

[27] Wilson LJ, Myat A, Sharma A, Maden M, Wingate RJ. Retinoic acid is a potential dorsalising signal in the late embryonic chick hindbrain. BMC Dev Biol. 2007,19;7:138.

[28] Chawla B, Swain W, Williams AL, Bohnsack BL. Retinoic Acid Maintains Function of Neural CrestDerived Ocular and Craniofacial Structures in Adult Zebrafish. Invest Ophthalmol Vis Sci. 2018,1;59(5):1924-1935.

[29] Duzyj CM, Paidas MJ, Jebailey L, Huang JS, Barnea ER. Prelmplantation factor (PIF*) promotes embryotrophic and neuroprotective decidual genes: effect negated by epidermal growth factor. $\mathrm{J}$ Neurodev Disord. 2014;6(1):36.

[30] An JH, Li FP, He P, Chen JS, Cai ZG, Liu SR, et al. Characteristics of Mesenchymal Stem Cells Isolated from the Bone Marrow of Red Pandas. Zoology (Jena). 2020;140:125775.

[31] Mukhamedshina YO, Gracheva OA, Mukhutdinova DM, Chelyshev YA, Rizvanov AA. Mesenchymal stem cells and the neuronal microenvironment in the area of spinal cord injury. Neural Regen Res. 2019;14(2):227-237.

[32] Jin W. Role of JAK/STAT3 Signaling in the Regulation of Metastasis, the Transition of Cancer Stem Cells, and Chemoresistance of Cancer by Epithelial-Mesenchymal Transition. Cells. 2020,15;9(1):217.

[33] MuhChyi C, Juliandi B, Matsuda T, Nakashima K. Epigenetic regulation of neural stem cell fate during corticogenesis. Int J Dev Neurosci. 2013;31(6):424-33.

[34] Lee N, Neitzel KL, Devlin BK, MacLennan AJ. STAT3 phosphorylation in injured axons before sensory and motor neuron nuclei: potential role for STAT3 as a retrograde signaling transcription factor. J Comp Neurol. 2004,5;474(4):535-45.

[35] Gao S, Durstine JL, Koh HJ, Carver WE, Frizzell N, Carson JA. Acute myotube protein synthesis regulation by IL-6-related cytokines. Am J Physiol Cell Physiol. 2017,1;313(5):C487-C500.

[36] Aberg MA, Ryttsén F, Hellgren G, Lindell K, Rosengren LE, MacLennan AJ, et al. Selective introduction of antisense oligonucleotides into single adult CNS progenitor cells using electroporation demonstrates the requirement of STAT3 activation for CNTF-induced gliogenesis. Mol Cell Neurosci. 2001;17(3):426-43.

[37] Su Y, Chen Z, Du H, Liu R, Wang W, Li H, et al. Silencing miR-21 induces polarization of astrocytes to the A2 phenotype and improves the formation of synapses by targeting glypican 6 via the signal transducer and activator of transcription-3 pathway after acute ischemic spinal cord injury. FASEB J. 2019;33(10):10859-10871. 
[38] Fathy M, Okabe M, M Othman E, Saad Eldien HM, Yoshida T. Preconditioning of Adipose-Derived Mesenchymal Stem-Like Cells with Eugenol Potentiates Their Migration and Proliferation In Vitro and Therapeutic Abilities in Rat Hepatic Fibrosis. Molecules. 2020,26;25(9):2020.

[39] Brick RM, Sun AX, Tuan RS. Neurotrophically Induced Mesenchymal Progenitor Cells Derived from Induced Pluripotent Stem Cells Enhance Neuritogenesis via Neurotrophin and Cytokine Production. Stem Cells Transl Med. 2018;7(1):45-58.

[40] Nicolaidou V, Wong MM, Redpath AN, Ersek A, Baban DF, Williams LM, et al. Monocytes induce STAT3 activation in human mesenchymal stem cells to promote osteoblast formation. PLoS One. 2012;7(7):e39871.

[41] Cosenza M, Civallero M, Fiorcari S, Pozzi S, Marcheselli L, Bari A, et al. The histone deacetylase inhibitor romidepsin synergizes with lenalidomide and enhances tumor cell death in T-cell lymphoma cell lines. Cancer Biol Ther. 2016,2;17(10):1094-1106.

[42] Wu M, Song D, Li H, Yang Y, Ma X, Deng S, et al. Negative regulators of STAT3 signaling pathway in cancers. Cancer Manag Res. 2019,29;11:4957-4969.

[43] Nakashima K, Yanagisawa M, Arakawa H, Kimura N, Hisatsune T, Kawabata M, et al. Synergistic signaling in fetal brain by STAT3-Smad1 complex bridged by p300. Science. 1999,16;284(5413):479-82.

[44] März P, Ozbek S, Fischer M, Voltz N, Otten U, Rose-John S. Differential response of neuronal cells to a fusion protein of ciliary neurotrophic factor/soluble CNTF-receptor and leukemia inhibitory factor. Eur $\mathrm{J}$ Biochem.2002;269(12):3023-31.

[45] Kober AM, Legewie S, Pforr C, Fricker N, Eils R, Krammer PH, et al. Caspase-8 activity has an essential role in CD95/Fas-mediated MAPK activation. Cell Death Dis. 2011,6;2(10):e212.

[46] Inman GJ, Allday MJ. Apoptosis induced by TGF-beta 1 in Burkitt's lymphoma cells is caspase 8 dependent but is death receptor independent. J Immunol. 2000,1;165(5):2500-10.

\section{Figures}


A
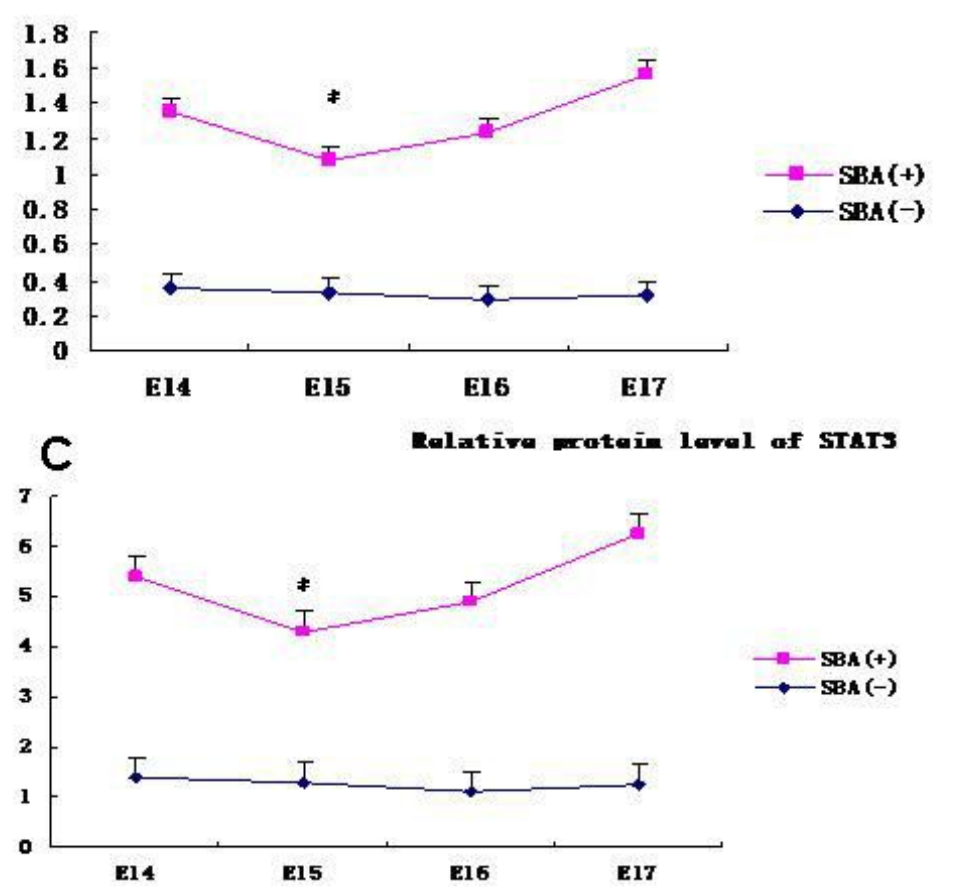

B

Relative and level of caspase 8

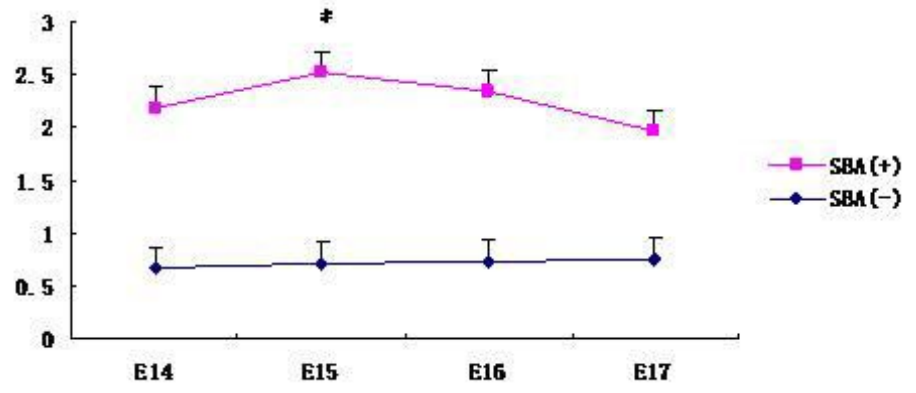

D Relative protein level of caspase 8

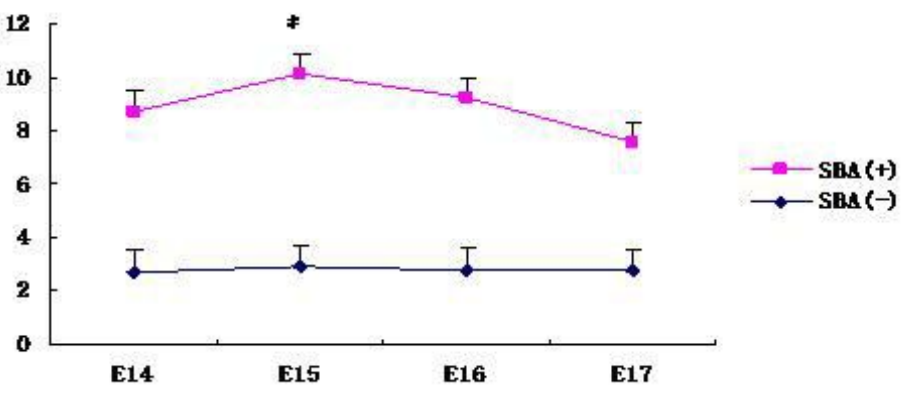

E $\overbrace{}^{\text {E14 }} \overbrace{\text { E15 }}^{\text {E17 }}$

SBA(-) SBA(+) SBA(-) SBA(+) SBA(-) SBA(+) SBA(-) SBA(+)

caspase 8

STAT3

$\beta$-actin

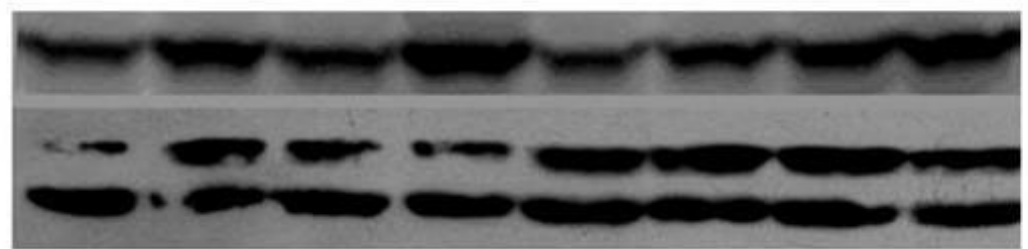

Figure 1

STAT3 and caspase-8 expression on 14, 15, 16 and 17 day in spinal cord of rat fetuses with control group and spina bifida aperta group. (A) and (B) mRNA level; (C), (D) and (E) protein level. 


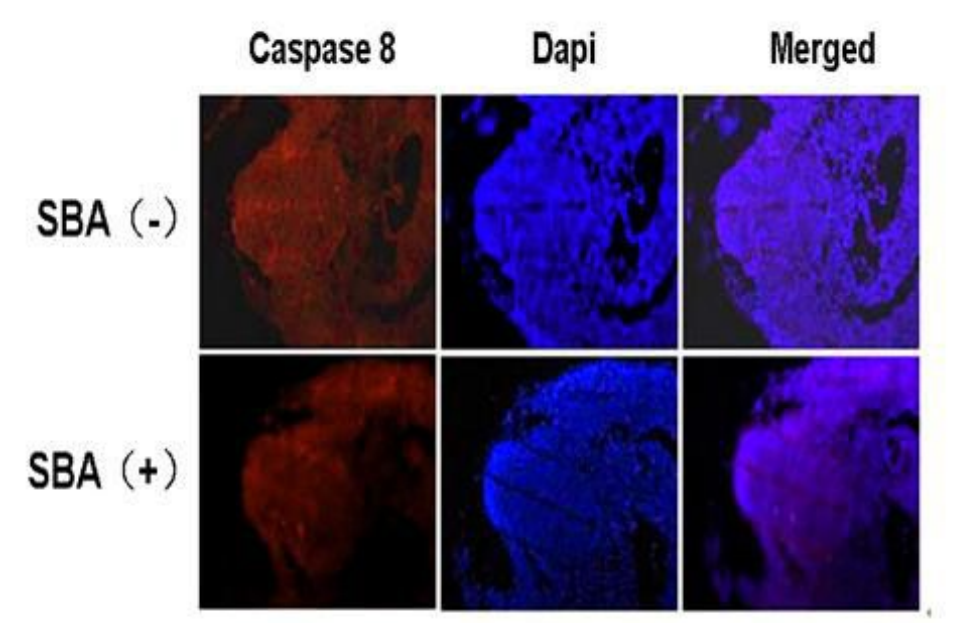

E14

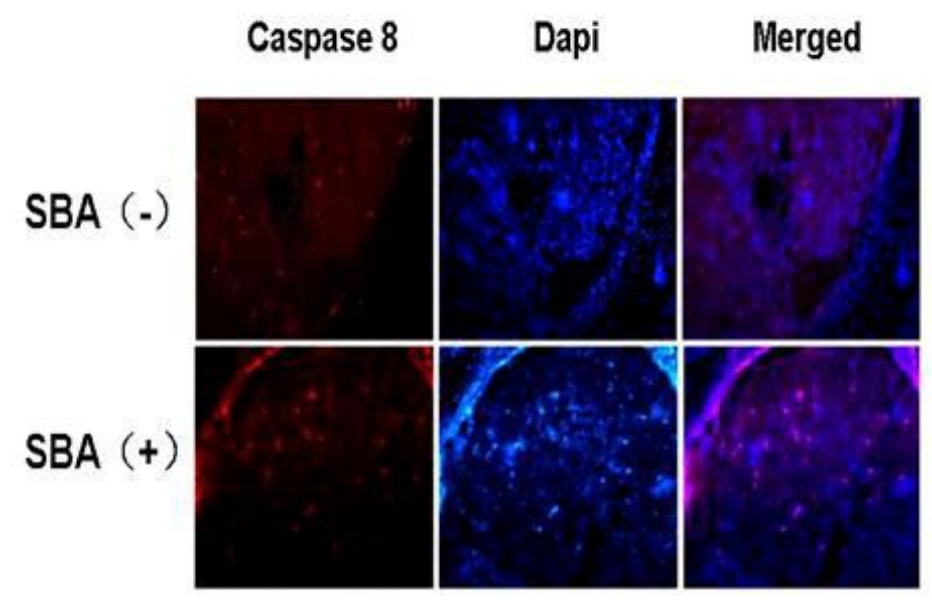

E16

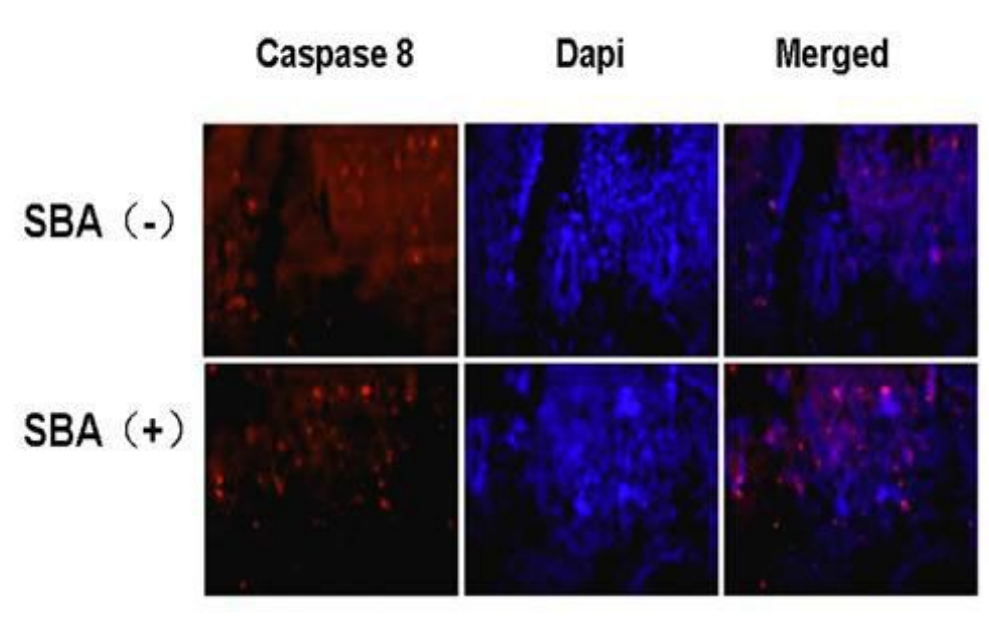

E15

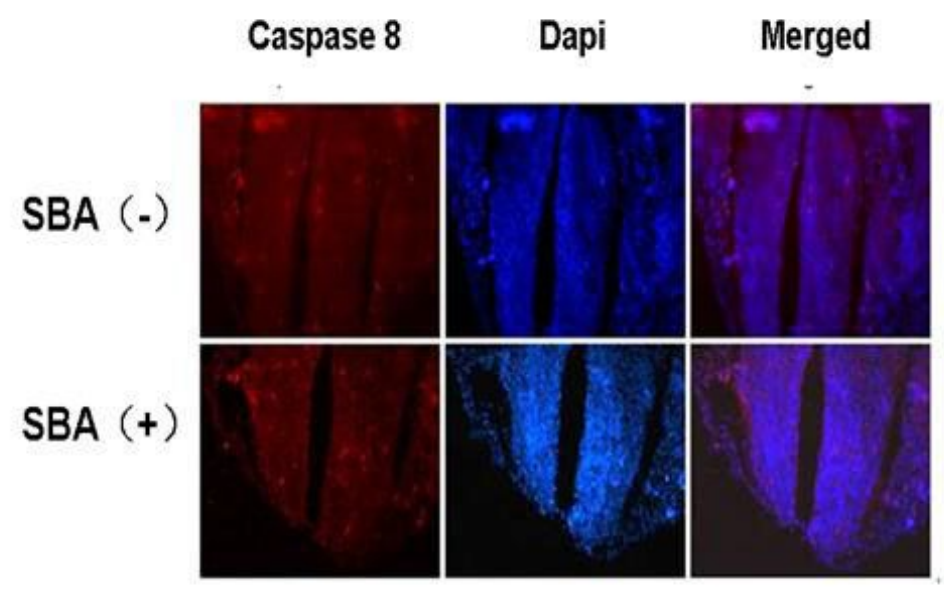

E17

Figure 2

Caspase-8 positive cells on 14, 15, 16 and 17 day in spinal cord of rat fetuses with control group and spina bifida aperta group by immunofluorescence. 


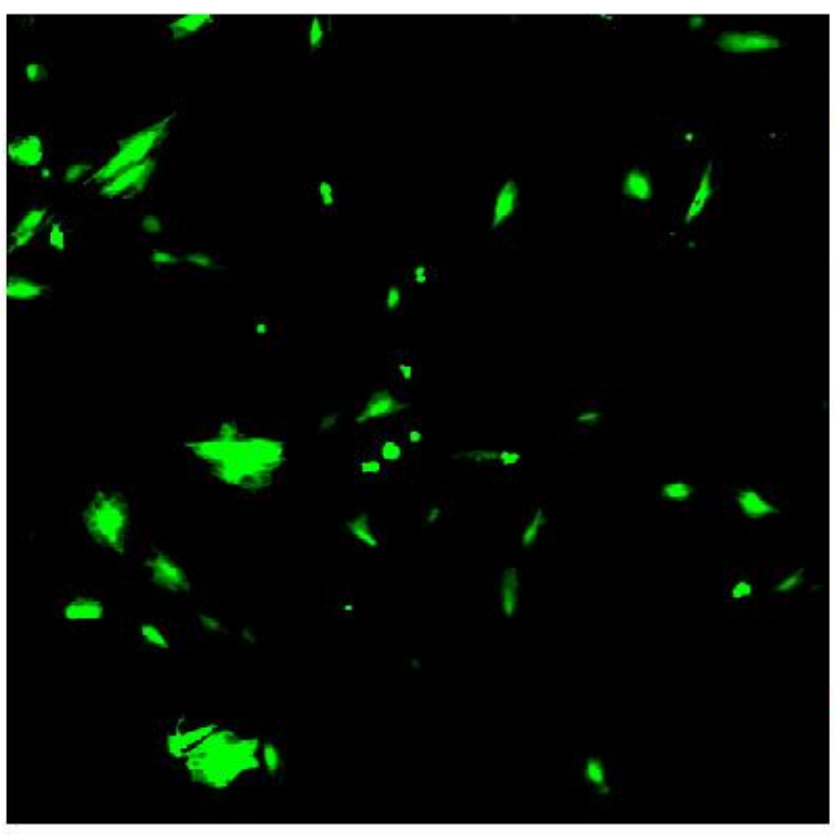

MSCs without STAT3

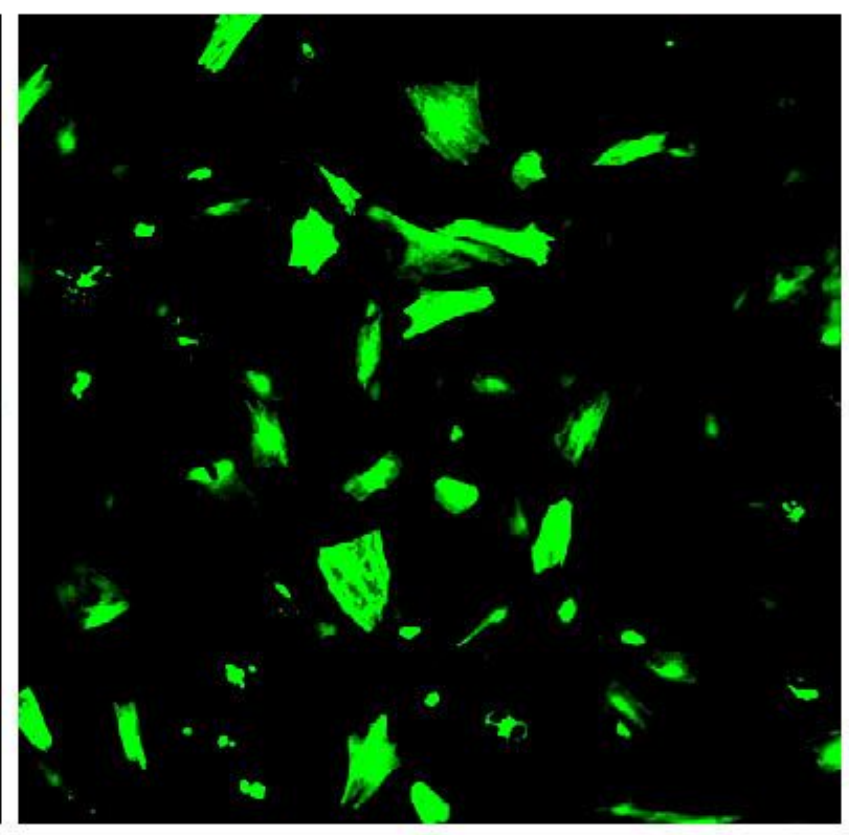

MSCs combined with STAT3

transfection before transplantation transfection before transplantation

Figure 3

The comparison between MSCs combined with STAT3 transfection and without transfection. Cell growth, proliferation and differentiation morphology are greater diversity. 


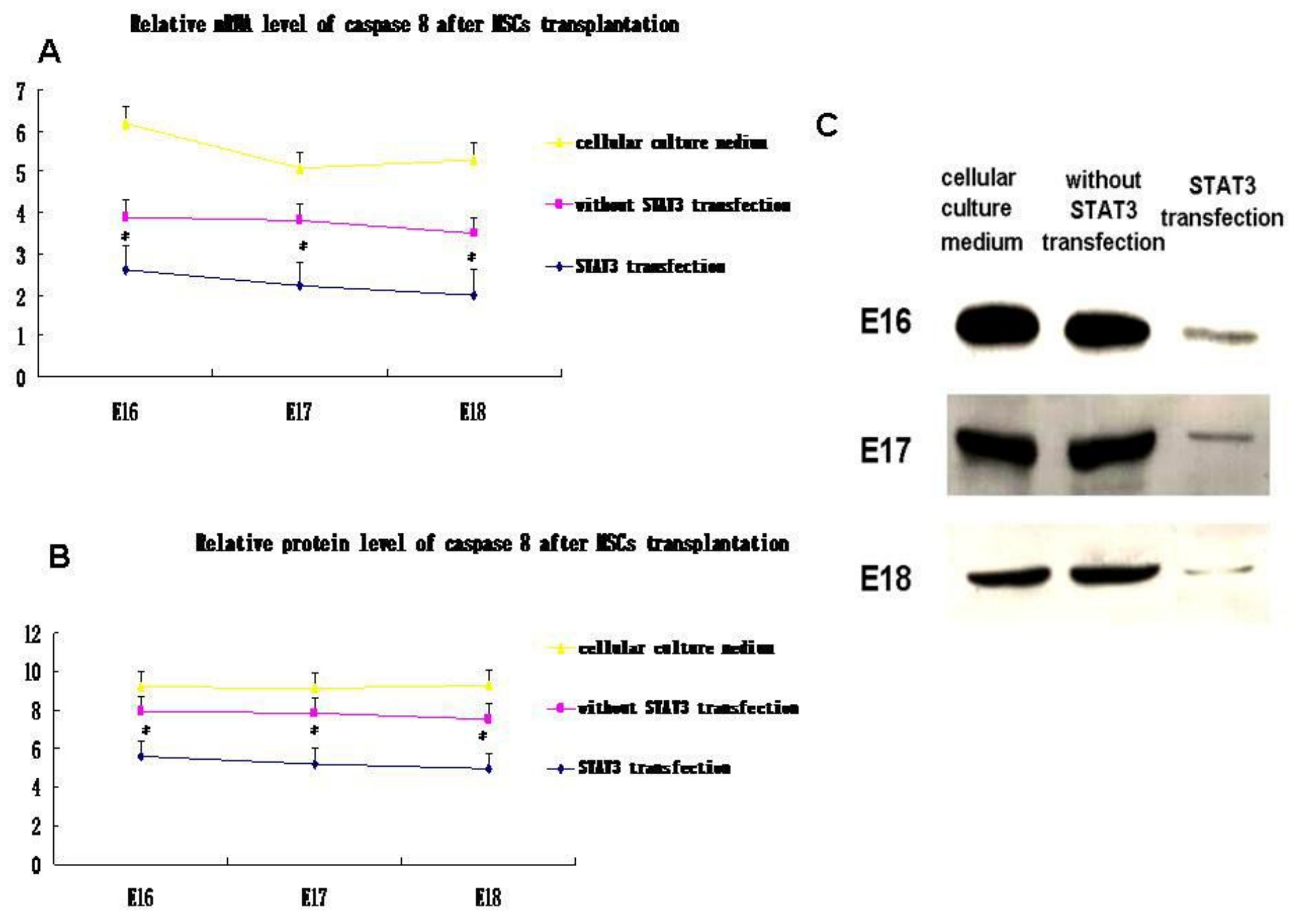

Figure 4

Caspase-8 expression in SBA transplantation with cellular culture medium, MSCs with STAT3 transfection and non-transfection group. (A) mRNA level; (B) and (C) protein level. 